\title{
Nonlinear ion-acoustic solitons in a magnetized quantum plasma with arbitrary degeneracy of electrons
}

\author{
Fernando Haas* \\ Physics Institute, Federal University of Rio Grande do Sul, CEP 91501-970, Avenida Bento Gonçalves 9500, Porto Alegre, RS, Brazil \\ Shahzad Mahmood \\ Theoretical Physics Division (TPD), PINSTECH, P.O. Nilore, Islamabad 44000, Pakistan \\ (Received 31 May 2016; revised manuscript received 25 August 2016; published 27 September 2016)
}

\begin{abstract}
Nonlinear ion-acoustic waves are analyzed in a nonrelativistic magnetized quantum plasma with arbitrary degeneracy of electrons. Quantum statistics is taken into account by means of the equation of state for ideal fermions at arbitrary temperature. Quantum diffraction is described by a modified Bohm potential consistent with finite-temperature quantum kinetic theory in the long-wavelength limit. The dispersion relation of the obliquely propagating electrostatic waves in magnetized quantum plasma with arbitrary degeneracy of electrons is obtained. Using the reductive perturbation method, the corresponding Zakharov-Kuznetsov equation is derived, describing obliquely propagating two-dimensional ion-acoustic solitons in a magnetized quantum plasma with degenerate electrons having an arbitrary electron temperature. It is found that in the dilute plasma case only electrostatic potential hump structures are possible, while in dense quantum plasma, in principle, both hump and dip soliton structures are obtainable, depending on the electron plasma density and its temperature. The results are validated by comparison with the quantum hydrodynamic model including electron inertia and magnetization effects. Suitable physical parameters for observations are identified.
\end{abstract}

DOI: 10.1103/PhysRevE.94.033212

\section{INTRODUCTION}

The ion-acoustic wave, which is the fundamental lowfrequency mode of plasma physics, is a prime focus of many current studies of localized electrostatic disturbances in laboratory, space, and astrophysical plasmas. The study of ion-acoustic waves has also gained importance in quantum plasmas for understanding electrostatic wave propagation in microscopic scales. During the last decade, there has been renewed interest in study of the collective wave phenomenon in quantum plasma, motivated by applications in semiconductors [1], high-intensity laser-plasma experiments [2-4], and highdensity astrophysical plasmas such as in the interior of massive planets and white dwarfs, neutron stars, or magnetars [5-7]. Quantum or degeneracy effects appear in plasmas when the de Broglie wavelength associated with the charged carriers becomes of the order of the interparticle distances. Quantum effects in plasmas are more frequently due to electrons, which are lighter than ions, and they include both Pauli's exclusion principle (for half-spin particles) and Heisenberg's uncertainty principle, due to the wavelike nature of the particles.

Quantum ion-acoustic waves in unmagnetized dense plasma have been investigated using quantum hydrodynamic models [8]. In quantum hydrodynamics, the momentum equation for degenerate electrons contains a pressure term compatible with a Fermi-Dirac distribution function, while the Bohm potential term is included to account for quantum diffraction [9-11]. Later on the quantum hydrodynamics model for plasmas was extended to include magnetic fields, with the associated quantum magnetohydrodynamics theory developed and discussed in connection with astrophysical dense plasmas [12]. Quantum Trivelpiece-Gould modes in a

*Corresponding author: fernando.haas@ufrgs.br dense magnetized quantum plasma were derived [13]. The exchange effects on low-frequency excitations in plasma have been discussed [14], using a modified Vlasov equation incorporating the exchange interaction $[15,16]$.

The Zakharov-Kuznetsov (ZK) equation was derived in 1974 , to study nonlinear propagation of ion-acoustic waves in magnetized plasmas [17-19]. The ZK equation is a multidimensional extension of the well-known Korteweg-de Vries equation for studying solitons (or single-pulse structures). In the degenerate magnetized plasma case, the cold Fermi electron gas assumption has been applied in the derivation of the appropriate ZK equation [20-22], restricted to the fully degenerate case of a negligible thermodynamic temperature in comparison to the Fermi temperature.

Linear ion-acoustic and electron Langmuir waves in a plasma with arbitrary degeneracy of electrons were studied using quantum kinetic theory [23]. The nonlinear theory of the isothermal ion-acoustic waves in degenerate unmagnetized electron plasmas was investigated [24]. The ranges of the phase velocities of the periodic ion-acoustic waves and the soliton speed were determined in degenerate plasma, but ignoring quantum diffraction effects. Also, nonlinear Langmuir waves in a dense plasma with arbitrary degeneracy of electrons in the absence as well as in the presence of quantum diffraction effects in the model have been studied [25]. Eliasson and Shukla [26] derived certain nonlinear quantum electron fluid equation by taking into account the moments of the Wigner equation and using the Fermi-Dirac distribution function for electrons of arbitrary temperature. The relativistic description of localized wave packets in electrostatic plasma [27] as well as the associated $\mathrm{ZK}$ equation for dense relativistic plasma [28] was obtained, in the limit of a negligible thermodynamic temperature. Recently, the hydrodynamic equations for ionacoustic excitations in electrostatic quantum plasma with arbitrary degeneracy were put forward [29]. The purpose 
of the present paper is to achieve a notable generalization of this work, obtaining the corresponding fluid theory for quantum magnetized ion-acoustic waves (MIAWs), in both the linear and the nonlinear realms. The extension is definitely of interest since magnetized degenerate plasmas are ubiquitous in astrophysics as well as in laboratory [30]. Specifically, it is of fundamental interest to access the nonlinear aspects of quantum MIAWs, which is a more accessible trend using hydrodynamic methods.

The paper is organized in the following way. In Sec. II, the set of dynamic equations for studying ion-acoustic waves in magnetized quantum plasmas with arbitrary degeneracy of electrons is presented. In Sec. III, the dispersion relation of obliquely propagating electrostatic linear waves in magnetized quantum plasma with arbitrary degeneracy of electrons is obtained. The limiting cases of waves parallel or perpendicular to the magnetic field are discussed, as well as the strongly magnetized ion limit. Section IV describes the modifications of the linear dispersion relation due to the inclusion of electron inertia and magnetization effects. Section V shows that the fluid theory is the limit case of quantum kinetic theory in the longwavelength limit, as it should be. In Sec. VI, using reductive perturbation methods, the ZK equation for two-dimensional propagation of nonlinear ion-acoustic waves is derived for a magnetized degenerate electron plasma of arbitrary temperature. The soliton solution is presented. Section VII illustrates the results, using suitable plasma parameters for observations, within the applicability range of the model. Section VIII contains the summary of the conclusions. Finally, the Appendix reports a more detailed derivation of the static electronic response from quantum kinetic theory, necessary in Sec. VI.

\section{DYNAMIC EQUATIONS FOR MAGNETIZED QUANTUM FLUIDS}

Consider a quantum electron-ion plasma with arbitrary degeneracy of electrons, embedded in an external magnetic field $\mathbf{B}_{0}=B_{0} \hat{x}$ directed along the $x$ axis. In principle, the electrostatic wave is assumed to propagate obliquely to the external magnetic field in the $x y$ plane, i.e., $\nabla=\left(\partial_{x}, \partial_{y}, 0\right)$. In order to study quantum MIAWs, ions are taken to be inertial, while electrons are assumed to be inertialess. The set of dynamic equations for MIAWs in a quantum plasma with arbitrary degeneracy of electrons is described as follows.

The ion continuity equation is given by

$$
\frac{\partial n_{i}}{\partial t}+\frac{\partial}{\partial x}\left(n_{i} u_{i x}\right)+\frac{\partial}{\partial y}\left(n_{i} u_{i y}\right)=0,
$$

while the ion momentum equations in component form are

$$
\begin{aligned}
\frac{\partial u_{i x}}{\partial t}+\left(u_{i x} \frac{\partial}{\partial x}+u_{i y} \frac{\partial}{\partial y}\right) u_{i x} & =-\frac{e}{m_{i}} \frac{\partial \phi}{\partial x} \\
\frac{\partial u_{i y}}{\partial t}+\left(u_{i x} \frac{\partial}{\partial x}+u_{i y} \frac{\partial}{\partial y}\right) u_{i y} & =-\frac{e}{m_{i}} \frac{\partial \phi}{\partial y}+\omega_{c i} u_{i z} \\
\frac{\partial u_{i z}}{\partial t}+\left(u_{i x} \frac{\partial}{\partial x}+u_{i y} \frac{\partial}{\partial y}\right) u_{i z} & =-\omega_{c i} u_{i y} .
\end{aligned}
$$

The momentum equation for the inertialess quantum electron fluid is

$0=-\frac{\nabla p}{n_{e}}+e \nabla \phi+\frac{\alpha \hbar^{2}}{6 m_{e}} \nabla\left[\frac{1}{\sqrt{n_{e}}}\left(\frac{\partial^{2}}{\partial x^{2}}+\frac{\partial^{2}}{\partial y^{2}}\right) \sqrt{n_{e}}\right]$.

The Poisson equation is written as

$$
\left(\frac{\partial^{2}}{\partial x^{2}}+\frac{\partial^{2}}{\partial y^{2}}\right) \phi=\frac{e}{\varepsilon_{0}}\left(n_{e}-n_{i}\right)
$$

where $\phi$ is the electrostatic potential. The ion fluid density and velocity are represented by $n_{i}$ and $\mathbf{u}_{i}=\left(u_{i x}, u_{i y}, u_{i z}\right)$, respectively, while $n_{e}$ is the electron fluid density. Also, $m_{e}$ and $m_{i}$ are the electron and ion masses, $-e$ is the electronic charge, $\varepsilon_{0}$ is the vacuum permittivity, $\hbar$ is the reduced Planck's constant, and $\omega_{c i}=e B_{0} / m_{i}$ is the ion cyclotron frequency. In equilibrium, we have $n_{e 0}=n_{i 0} \equiv n_{0}$. The electron's fluid pressure $p=p\left(n_{e}\right)$ is specified by a barotropic equation of state which is given below. The last term on the right-hand side of the momentum Eq. (5), for electrons is the quantum force, which arises from the Bohm potential, giving rise to quantum diffraction or tunneling effects due to the wavelike nature of the charged particles. The dimensionless quantity $\alpha$ is selected in order to fit the kinetic linear dispersion relation in the long-wavelength limit, in a Fermi-Dirac equilibrium, as shown in the continuation. Quantum effects on ions are ignored in view of their large mass in comparison to electrons. In addition, temperature effects on ions are disregarded. Finally, to avoid too much complexity and to focus on the interplay between degeneracy and quantum recoil, exchange effects are also ignored.

The equation of state can be obtained from the moments of a local Fermi-Dirac distribution function [29,31] of an ideal Fermi gas and reads

$$
p=\frac{n_{e}}{\beta} \frac{\mathrm{Li}_{5 / 2}\left(-e^{\beta \mu}\right)}{\operatorname{Li}_{3 / 2}\left(-e^{\beta \mu}\right)},
$$

where $\beta=\left(\kappa_{B} T\right)^{-1}, \kappa_{B}$ is the Boltzmann constant, $T$ is the temperature, and $\mu$ is the chemical potential, satisfying

$$
n_{e}=n_{0} \frac{\mathrm{Li}_{3 / 2}\left(-e^{\beta \mu}\right)}{\mathrm{Li}_{3 / 2}\left(-e^{\beta \mu_{0}}\right)} .
$$

The equilibrium chemical potential $\mu_{0}$ is related to the equilibrium density $n_{0}$ through

$$
-\frac{n_{0}}{\mathrm{Li}_{3 / 2}\left(-e^{\beta \mu_{0}}\right)}\left(\frac{\beta m_{e}}{2 \pi}\right)^{3 / 2}=2\left(\frac{m_{e}}{2 \pi \hbar}\right)^{3}=\mathcal{A},
$$

where the quantity $\mathcal{A}$ was defined for later convenience.

Equations (7) and (8) contain the polylogarithm function $\operatorname{Li}_{v}(-z)$ with index $v$, which for $v>0$ can be defined [32] as

$$
\operatorname{Li}_{v}(-z)=-\frac{1}{\Gamma(v)} \int_{0}^{\infty} \frac{s^{\nu-1}}{1+e^{s} / z} d s, \quad v>0
$$

where $\Gamma(v)$ is the gamma function. For $v<0$ one applies

$$
\operatorname{Li}_{v}(-z)=\left(z \frac{\partial}{\partial z}\right) \operatorname{Li}_{v+1}(-z)
$$

as many times as necessary, where $v+1>0$. 


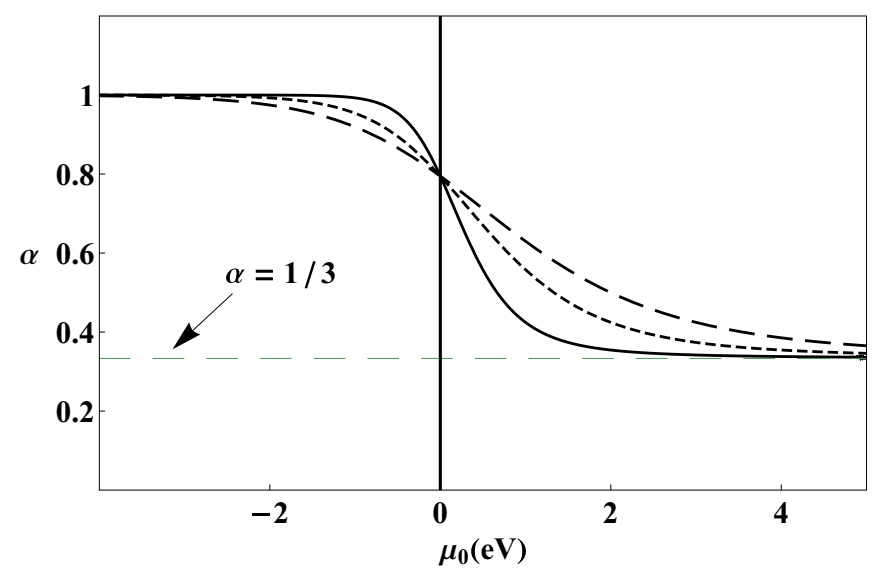

FIG. 1. Coefficient $\alpha$ from Eq. (12), as a function of the chemical potential $\mu_{0}$. Solid line: $T=4000 \mathrm{~K}$. Dotted line: $T=6000 \mathrm{~K}$. Dashed line: $T=8000 \mathrm{~K}$.

The numerical coefficient $\alpha$ appearing in the Bohm potential term in Eq. (5) has been derived from finite-temperature quantum kinetic theory for low-frequency electrostatic excitations in [29],

$$
\alpha=\frac{\operatorname{Li}_{3 / 2}\left(-e^{\beta \mu_{0}}\right) \operatorname{Li}_{-1 / 2}\left(-e^{\beta \mu_{0}}\right)}{\left[\operatorname{Li}_{1 / 2}\left(-e^{\beta \mu_{0}}\right)\right]^{2}},
$$

expressed as a function of the equilibrium fugacity $z=$ $\exp \left(\beta \mu_{0}\right)$. The treatment of [29] considered nonmagnetized plasmas, but in Sec. V it is proved that Eq. (12) applies to MIAWs too. As discussed in [29], in the classical limit $(z \ll 1)$ one has $\alpha \approx 1$, while in the full degenerate limit $(z \gg 1)$ one has $\alpha \approx 1 / 3$. The same behavior is seen for $\alpha$ as a function of the chemical potential, as depicted in Fig. 1, showing a transition zone from classical to dense regimes.

It happens [33] that the finite-temperature quantum hydrodynamic equations using $\alpha$ from Eq. (12) are consistent with the results from orbital free density functional theory $[34,35]$.

\section{LINEAR WAVES}

In order to find the dispersion relation for electrostatic wave in a magnetized quantum plasma with arbitrary degeneracy of electrons, we linearize the system of Eqs. (1)-(6) by considering

$$
\begin{aligned}
n_{i} & =n_{0}+n_{i 1}, \quad n_{e}=n_{0}+n_{e 1}, \quad u_{i x}=u_{i x 1}, \\
u_{i y} & =u_{i y 1}, \quad u_{i z}=u_{i z 1}, \quad \phi=\phi_{1},
\end{aligned}
$$

inducing a correction $\mu=\mu_{0}+\mu_{1}$, where the subscript 1 denotes first-order quantities. In particular, using the expansion of the polylogarithm function to first order, i.e.,

$$
\operatorname{Li}_{v}\left(-e^{\beta\left(\mu_{0}+\mu_{1}\right)}\right)=\operatorname{Li}_{v}\left(-e^{\beta \mu_{0}}\right)+\beta \mu_{1} \operatorname{Li}_{v-1}\left(-e^{\beta \mu_{0}}\right),
$$

and considering plane-wave perturbations $\sim \exp \left[i\left(k_{x} x+\right.\right.$ $\left.k_{y} y-\omega t\right)$, the result is

$$
1+\chi_{i}(\omega, \mathbf{k})+\chi_{e}(0, \mathbf{k})=0,
$$

where the ionic and electronic susceptibilities are given by, respectively,

$$
\begin{aligned}
& \chi_{i}(\omega, \mathbf{k})=-\frac{\omega_{p i}^{2}\left(\omega^{2}-\omega_{c i}^{2} \cos ^{2} \theta\right)}{\omega^{2}\left(\omega^{2}-\omega_{c i}^{2}\right)}, \\
& \chi_{e}(0, \mathbf{k})=\omega_{p e}^{2}\left[\frac{1}{m_{e}}\left(\frac{d p}{d n_{e}}\right)_{0} k^{2}+\frac{\alpha \hbar^{2} k^{4}}{12 m_{e}^{2}}\right]^{-1},
\end{aligned}
$$

where $\omega_{p j}^{2}=n_{0} e^{2} /\left(m_{j} \varepsilon_{0}\right)$ for $j=i, e$ and $\mathbf{k}=k(\cos \theta$, $\sin \theta, 0)$. Due to the neglect of electron's inertia, only the static electronic susceptibility $\chi_{e}(0, \mathbf{k})$ is necessary. There is no loss of generality in assuming waves in the $x y$ plane, due to the cylindrical geometry around the $x$ axis.

The dispersion relation (15), develops as a quadratic equation for $\omega^{2}$ whose solution is

$\omega^{2}=\frac{1}{2}\left[\omega_{0}^{2}+\omega_{c i}^{2} \pm\left(\left(\omega_{0}^{2}+\omega_{c i}^{2}\right)^{2}-4 \omega_{0}^{2} \omega_{c i}^{2} \cos ^{2} \theta\right)^{1 / 2}\right]$,

where $\omega_{0}$ was already obtained [29] in the case of unmagnetized quantum ion-acoustic waves,

$$
\omega_{0}^{2}=\frac{c_{s}^{2} k^{2}\left[1+H^{2}\left(k \lambda_{D}\right)^{2} / 4\right]}{1+\left(k \lambda_{D}\right)^{2}+H^{2}\left(k \lambda_{D}\right)^{4} / 4} .
$$

In Eq. (19) one has the ion-acoustic speed $c_{s}$ which follows from

$$
c_{s}^{2}=\frac{1}{m_{i}}\left(\frac{d p}{d n_{e}}\right)_{0}=\frac{\kappa_{B} T}{m_{i}} \frac{\mathrm{Li}_{3 / 2}\left(-e^{\beta \mu_{0}}\right)}{\mathrm{Li}_{1 / 2}\left(-e^{\beta \mu_{0}}\right)},
$$

the generalized electronic screening length $\lambda_{D}$ from

$$
\lambda_{D}^{2}=\frac{c_{s}^{2}}{\omega_{p i}^{2}}=\frac{\kappa_{B} T}{m_{e} \omega_{p e}^{2}} \frac{\mathrm{Li}_{3 / 2}\left(-e^{\beta \mu_{0}}\right)}{\mathrm{Li}_{1 / 2}\left(-e^{\beta \mu_{0}}\right)},
$$

and the quantum diffraction parameter $H$ specified by

$$
H=\frac{\beta \hbar \omega_{p e}}{\sqrt{3}}\left(\frac{\mathrm{Li}_{-1 / 2}\left(-e^{\beta \mu_{0}}\right)}{\mathrm{Li}_{3 / 2}\left(-e^{\beta \mu_{0}}\right)}\right)^{1 / 2} .
$$

In the dilute plasma limit $e^{\beta \mu_{0}} \ll 1$, implying $\operatorname{Li}_{v}\left(-e^{\beta \mu_{0}}\right) \approx$ $-e^{\beta \mu_{0}}$, one has $c_{s} \approx \sqrt{\kappa_{B} T / m_{i}}$ and $\lambda_{D}=\sqrt{\kappa_{B} T /\left(m_{e} \omega_{p e}^{2}\right)}$, which, respectively, are the more traditional ion-acoustic speed and Debye length, and $H \approx \beta \hbar \omega_{p e} / \sqrt{3}$. On the other hand, in the fully degenerate case $e^{\beta \mu_{0}} \gg 1$, using $\operatorname{Li}_{\nu}\left(-e^{\beta \mu_{0}}\right) \approx$ $-\left(\beta \mu_{0}\right)^{v} / \Gamma(v+1)$ one has $\mu_{0} \approx E_{F}=\hbar^{2}\left(3 \pi^{2} n_{0}\right)^{2 / 3} /\left(2 m_{e}\right)$, which is the Fermi energy, and $c_{s} \approx \sqrt{(2 / 3) E_{F} / m_{i}}$ and $\lambda_{D}=$ $\sqrt{2 E_{F} /\left(3 m_{e} \omega_{p e}^{2}\right)}$, which are, respectively, the quantum ionacoustic speed and the Thomas-Fermi screening length, and $H \approx(1 / 2) \hbar \omega_{p e} / E_{F}$. The dispersion relation (18), is formally the same as for classical magnetized plasma [36,37], provided the fully quantum ion-acoustic frequency $\omega_{0}$ is replaced by its purely classical counterpart.

As is apparent from the dispersion relation (15), ions are responsible for providing inertia effects, while electrons are responsible for the kinetic energy (arising from the standard, thermodynamic temperature and/or Fermi pressure) and quantum diffraction is represented by the parameter $H$. It is convenient to rewrite Eq. (22) using Eq. (9), yielding

$$
H^{2}=-\frac{2 \alpha_{F}}{3} \sqrt{\frac{2 \beta m_{e} c^{2}}{\pi}} \operatorname{Li}_{-1 / 2}\left(-e^{\beta \mu_{0}}\right),
$$




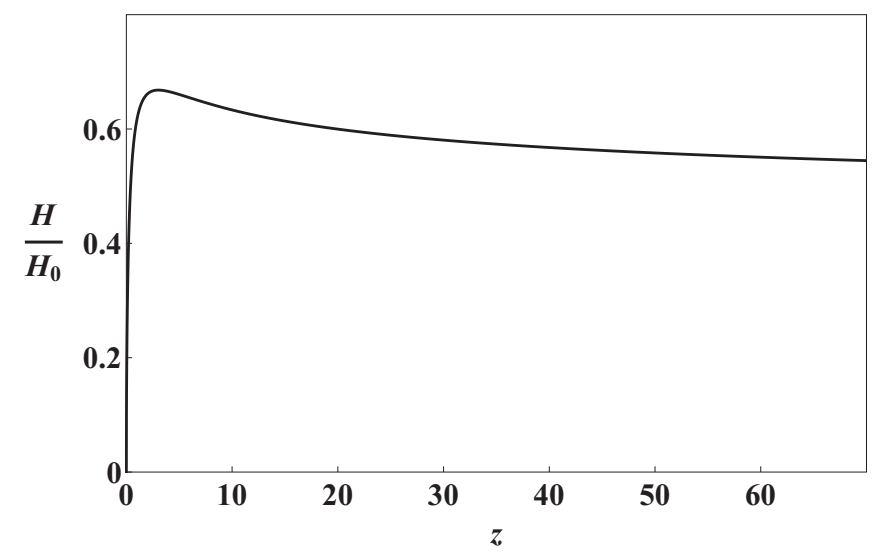

FIG. 2. Quantum diffraction parameter defined in Eq. (23), as a function of the equilibrium fugacity $z=e^{\beta \mu_{0}}$, and normalized to $H_{0}=\left[\left(2 \alpha_{F} / 3\right)\left(2 \beta m_{e} c^{2} / \pi\right)^{1 / 2}\right]^{1 / 2}$.

where $\alpha_{F}=e^{2} /\left(4 \pi \varepsilon_{0} \hbar c\right) \approx 1 / 137$ is the fine-structure constant. Obviously the theory is nonrelativistic, in spite of the appearance of the rest energy $m_{e} c^{2}$ in Eq. (23).

For a fixed temperature, $H$ is a simple function of the fugacity $z=\exp \left(\beta \mu_{0}\right)$, as shown in Fig. 2. It is seen that the pure wavelike quantum effects are enhanced for higher densities (and fugacities) up to $z \approx 3.03$, while for larger degeneracy the quantum statistical effects prevail, showing that the quantum force becomes less effective in denser systems, in view of Pauli's exclusion principle. Therefore for dilute systems $H$ increases with the density and decreases with the temperature, while for fully degenerate systems the leadingorder behavior shows a decrease in $H$ with increasing density.

The positive sign in Eq. (18) corresponds to fast electrostatic waves, and a negative sign corresponds to slow electrostatic waves in a magnetized plasma. The effect of degeneracy for arbitrary angle $\theta$ is not entirely straightforward to identify, due to the somewhat involved expression (18). However, in practical applications it is likely to have $\omega_{0} \gg \omega_{c i}$, so that the fast mode becomes $\omega^{2} \approx \omega_{0}^{2}+\omega_{c i}^{2} \sin ^{2} \theta$, while the slow mode becomes $\omega^{2} \approx \omega_{c i}^{2} \cos ^{2} \theta$. Since quantum effects are present only on $\omega_{0}^{2} \gg \omega_{c i}^{2}$, it happens that the fast wave has an angular dependence appearing as a correction, while the slow wave is strongly angle dependent but not as influenced by quantum effects. As an example, consider hydrogen plasma with an ambient magnetic field $B_{0}=10^{3} \mathrm{~T}$, yielding the ion cyclotron frequency $\omega_{c i}=9.58 \times 10^{10} \mathrm{rad} / \mathrm{s}$. For solid-density plasma with $T=10^{6} \mathrm{~K}, n_{0}=5 \times 10^{30} \mathrm{~m}^{-3}$, and a wave- umber $k=$ $2 \pi \times 10^{9} \mathrm{~m}^{-1}$, one has $\omega_{0}=6.50 \times 10^{14} \mathrm{rad} / \mathrm{s} \gg \omega_{c i}$. These parameters are in accordance with the more detailed validity conditions discussed in Sec. VII.

In addition, some significant limiting cases are described below.

\section{A. Wave propagation parallel to the magnetic field}

Considering $\theta=0$ in Eq. (18), one has either $\omega=\omega_{c i}$ (the ion cyclotron frequency) or $\omega=\omega_{0}$. The latter has been discussed in [29]. In the limit $k \lambda_{D} \ll 1$ one has $\omega_{0} \approx c_{s} k$, where the ion-acoustic speed contains only quantum degeneracy effects as seen from Eq. (20).

\section{B. Wave propagation perpendicular to the magnetic field}

Considering $\theta=\pi / 2$ in Eq. (18), one has a vanishing solution $\left(\omega^{2}=0\right)$ as well as a quantum modified electrostatic ion cyclotron wave given by $\omega^{2}=\omega_{c i}^{2}+\omega_{0}^{2}$.

\section{Strongly magnetized ions}

For completeness we consider the strongly magnetized ion case. If $\omega_{c i} \gg \omega_{0}$, one has the fast mode,

$$
\omega^{2}=\omega_{c i}^{2}\left[1+\frac{\omega_{0}^{2}}{\omega_{c i}^{2}} \sin ^{2} \theta+\frac{\omega_{0}^{4}}{4 \omega_{c i}^{4}} \sin ^{2}(2 \theta)+O\left(\left(\frac{\omega_{0}}{\omega_{c i}}\right)^{6}\right)\right],
$$

and the slow mode,

$$
\omega^{2}=\omega_{0}^{2} \cos ^{2} \theta\left[1-\frac{\omega_{0}^{2}}{\omega_{c i}^{2}} \sin ^{2} \theta+O\left(\left(\frac{\omega_{0}}{\omega_{c i}}\right)^{4}\right)\right] .
$$

\section{ELECTRON INERTIA AND MAGNETIZATION EFFECTS}

In the purely classical case, the conditions of applicability of the model are well known [18,19]. In the quantum case, it is interesting to include electron inertia and magnetization effects, to measure the limitations of Eq. (15). In this context one adds to Eqs. (1)-(4) and (6) the electron continuity equation,

$$
\frac{\partial n_{e}}{\partial t}+\frac{\partial}{\partial x}\left(n_{e} u_{e x}\right)+\frac{\partial}{\partial y}\left(n_{e} u_{e y}\right)=0,
$$

and replaces Eq. (5) with

$$
\begin{aligned}
m_{e}\left(\frac{\partial \mathbf{u}_{e}}{\partial t}\right. & \left.+\mathbf{u}_{e} \cdot \nabla \mathbf{u}_{\mathbf{e}}\right)=-\frac{\nabla p}{n_{e}}-e\left(-\nabla \phi+\mathbf{u}_{e} \times \mathbf{B}_{0}\right) \\
& +\frac{\alpha \hbar^{2}}{6 m_{e}} \nabla\left[\frac{1}{\sqrt{n_{e}}}\left(\frac{\partial^{2}}{\partial x^{2}}+\frac{\partial^{2}}{\partial y^{2}}\right) \sqrt{n_{e}}\right],
\end{aligned}
$$

where $\mathbf{u}_{e}=\left(u_{e x}, u_{e y}, u_{e z}\right)$ is the electron fluid velocity. Proceeding as in Sec. III and also supposing linear perturbations where $\mathbf{u}_{e}=\mathbf{u}_{e 1}$, the result is

$$
1+\chi_{i}(\omega, \mathbf{k})+\chi_{e}(\omega, \mathbf{k})=0
$$

where the ionic susceptibility is still given by Eq. (16) and

$$
\chi_{e}(\omega, \mathbf{k})=-\frac{\omega_{p e}^{2}\left(\omega^{2}-\omega_{c e}^{2} \cos ^{2} \theta\right)}{\omega^{4}-\left(k^{2} v_{T}^{2}(k)+\omega_{c e}^{2}\right) \omega^{2}+k^{2} v_{T}^{2}(k) \omega_{c e}^{2} \cos ^{2} \theta},
$$

where $\omega_{c e}=e B_{0} / m_{e}$ is the electron cyclotron frequency and

$$
v_{T}^{2}(k)=\frac{1}{m_{e}}\left(\frac{d p}{d n_{e}}\right)_{0}+\frac{\alpha \hbar^{2} k^{2}}{12 m_{e}^{2}} .
$$

It is not the purpose of this work to develop the full consequences of the dispersion relation (28), but it is useful to observe that in the formal limit $\omega \rightarrow 0$ the electron response (29), regains the static electronic response $\chi_{e}(0, \mathbf{k})$ given by Eq. (17). Moreover, by inspection of Eq. (29) it is found that such a limit is attended for a warm electron fluid, where $k^{2} v_{T}^{2}(k) \gg \omega_{c e}^{2}$, so that the electrons' magnetization could be disregarded, and $k^{2} v_{T}^{2}(k) \gg \omega^{2}$, which is attainable for 
low-frequency excitations. In addition, note that $v_{T}(k)$ from Eq. (30) depends not only on the pressure but also on quantum diffraction effects. On the other hand, ions are assumed to be cold and nonquantum enough.

\section{COMPARISON TO KINETIC THEORY}

The results from hydrodynamics should agree with kinetic theory in the long-wavelength limit. Therefore it is necessary to compare the ionic and electronic responses found from kinetic theory to the susceptibilities shown in Eqs. (16) and (17). Since ions are safely assumed to be classical in most cases, their particle distribution function $f_{i}=f_{i}(\mathbf{r}, \mathbf{v}, t)$ satisfies Vlasov's equation, which presently is

$$
\left[\frac{\partial}{\partial t}+\mathbf{v} \cdot \nabla+\frac{e}{m_{i}}\left(-\nabla \phi+\mathbf{v} \times \mathbf{B}_{0}\right) \cdot \frac{\partial}{\partial \mathbf{v}}\right] f_{i}=0 .
$$

On the other hand, the quantum nature of electrons merits the use of the quantum Vlasov equation satisfied by the electronic Wigner quasidistribution $f_{e}=f_{e}(\mathbf{r}, \mathbf{v}, t)$,

$$
\begin{aligned}
\frac{\partial f_{e}}{\partial t} & +\mathbf{v} \cdot \nabla f_{e}-\frac{i e}{\hbar}\left(\frac{m_{e}}{2 \pi \hbar}\right)^{3} \\
& \times \int d \mathbf{s} d \mathbf{v}^{\prime} \exp \left(\frac{i m_{e}\left(\mathbf{v}^{\prime}-\mathbf{v}\right) \cdot \mathbf{s}}{\hbar}\right) \\
& \times\left[\phi\left(\mathbf{r}+\frac{\mathbf{s}}{2}, t\right)-\phi\left(\mathbf{r}-\frac{\mathbf{s}}{2}, t\right)\right] f_{e}\left(\mathbf{r}, \mathbf{v}^{\prime}, t\right)=0 .
\end{aligned}
$$

All integrals run from $-\infty$ to $\infty$, unless otherwise stated. Moreover, under the same assumption as before, namely, large electron thermal and quantum (statistical and diffraction) effects, the magnetic force on electrons was omitted in Eq. (32).

The scalar potential is self-consistently determined by Poisson's equation,

$$
\left(\frac{\partial^{2}}{\partial x^{2}}+\frac{\partial^{2}}{\partial y^{2}}\right) \phi=\frac{e}{\varepsilon_{0}}\left(\int f_{e} d \mathbf{v}-\int f_{i} d \mathbf{v}\right),
$$

where we take spatial variations in the $x y$ plane only.

Proceeding as in Sec. III, assuming plane-wave perturbations $\sim \exp \left[i\left(k_{x} x+k_{y} y-\omega t\right)\right]$ around isotropic in velocities equilibria, the dispersion relation $1+\chi_{i}(\omega, \mathbf{k})+\chi_{e}(\omega, \mathbf{k})=0$ is easily derived. Disregarding the negligibly small Landau damping of MIAWs in the case of cold ions, it is found [38,39] that the ionic susceptibility from kinetic theory coincides with the fluid expression (16). On the other hand, for low-frequency waves, the static limit $\chi_{e}(0, \mathbf{k})$ is sufficient for electrons, reading

$$
\chi_{e}(0, \mathbf{k})=\frac{e^{2}}{\varepsilon_{0} \hbar k^{2}} \int \frac{d \mathbf{v}}{\mathbf{k} \cdot \mathbf{v}}\left[F\left(\mathbf{v}-\frac{\hbar \mathbf{k}}{2 m_{e}}\right)-F\left(\mathbf{v}+\frac{\hbar \mathbf{k}}{2 m_{e}}\right)\right]
$$

where the principal value of the integral is understood if necessary and where the equilibrium electronic Wigner function is $f_{e}=F(\mathbf{v})$.

Consider a Fermi-Dirac equilibrium,

$$
F(\mathbf{v})=\frac{\mathcal{A}}{1+e^{\beta\left(m_{e} v^{2} / 2-\mu_{0}\right)}}, \quad v=|\mathbf{v}|,
$$

where the normalization constant $\mathcal{A}$ is given in Eq. (9), assuring that $\int F(\mathbf{v}) d \mathbf{v}=n_{0}$.

It turns out that the right-hand side of Eq. (34) can be evaluated as a power series of the quantum recoil $q=\sqrt{\beta /\left(2 m_{e}\right)} \hbar k / 2$, supposed to be a small quantity for long wavelengths and/or sufficiently high electronic temperature:

$$
\begin{aligned}
\chi_{e}(0, \mathbf{k})= & \frac{\beta m_{e} \omega_{p e}^{2}}{\sqrt{\pi} \mathrm{Li}_{3 / 2}(-z) k^{2}}\left[\Gamma\left(\frac{1}{2}\right) \mathrm{Li}_{1 / 2}(-z)\right. \\
& +\Gamma\left(-\frac{1}{2}\right) \mathrm{Li}_{-1 / 2}(-z) \frac{q^{2}}{3} \\
& \left.+\Gamma\left(-\frac{3}{2}\right) \mathrm{Li}_{-3 / 2}(-z) \frac{q^{4}}{5}+\cdots\right] \\
= & \frac{\beta m_{e} \omega_{p e}^{2}}{\sqrt{\pi} \mathrm{Li}_{3 / 2}(-z) k^{2}} \sum_{j=0}^{\infty} \Gamma\left(\frac{1}{2}-j\right) \\
& \times \operatorname{Li}_{1 / 2-j}(-z) \frac{q^{2 j}}{2 j+1},
\end{aligned}
$$

where $z=e^{\beta \mu_{0}}$. The derivation is detailed in the Appendix. Expression (36) is exact, as long as the series converges. Moreover, it coincides with the static limit of Eq. (29) in Ref. [40], where only the leading $O\left(q^{2}\right)$ quantum recoil correction was calculated.

For the sake of comparison, the hydrodynamic result from Eq. (17) can be rewritten as

$$
\begin{aligned}
& \chi_{e}(0, \mathbf{k}) \\
& =\frac{\beta m_{e} \omega_{p e}^{2} \mathrm{Li}_{1 / 2}(-z)}{\operatorname{Li}_{3 / 2}(-z) k^{2}}\left(1+\frac{2 q^{2}}{3} \frac{\mathrm{Li}_{-1 / 2}(-z)}{\mathrm{Li}_{1 / 2}(-z)}\right)^{-1} \\
& =\frac{\beta m_{e} \omega_{p e}^{2}}{\operatorname{Li}_{3 / 2}(-z) k^{2}}\left(\operatorname{Li}_{1 / 2}(-z)-\frac{2 q^{2}}{3} \mathrm{Li}_{-1 / 2}(-z)+O\left(q^{4}\right)\right),
\end{aligned}
$$

which coincides with Eq. (36) in the long-wavelength limit, as $\Gamma(1 / 2)=\sqrt{\pi}, \Gamma(-1 / 2)=-2 \sqrt{\pi}$. This completes the justification of $\alpha$ in Eq. (12) in the magnetized case.

\section{ZAKHAROV-KUZNETSOV EQUATION FOR ARBITRARY DEGENERACY}

In order to derive the ZK equation for obliquely propagating MIAWs in arbitrary degenerate plasma, it is convenient to make use of normalized quantities. The dispersion relation (18), suggests the use of the dimensionless variables $(\tilde{x}, \tilde{y})=$ $(x, y) / \lambda_{D} \tilde{t}=\omega_{p i} t,\left(\tilde{u}_{i x}, \tilde{u}_{i y}, \tilde{u}_{i z}\right)=\left(u_{i x}, u_{i y}, u_{i z}\right) / c_{s}$, and $\tilde{\phi}=$ $e \phi /\left(m_{i} c_{s}^{2}\right), \tilde{n}_{j}=n_{j} / n_{0}$, where $j=e, i$. Equations (1)-(6) are then written as

$$
\begin{array}{r}
\frac{\partial \tilde{n}_{i}}{\partial \tilde{t}}+\frac{\partial}{\partial \tilde{x}}\left(\tilde{n}_{i} \tilde{u}_{i x}\right)+\frac{\partial}{\partial \tilde{y}}\left(\tilde{n}_{i} \tilde{u}_{i y}\right)=0, \\
\frac{\partial \tilde{u}_{i x}}{\partial \tilde{t}}+\left(\tilde{u}_{i x} \frac{\partial}{\partial \tilde{x}}+\tilde{u}_{i y} \frac{\partial}{\partial \tilde{y}}\right) \tilde{u}_{i x}=-\frac{\partial \tilde{\phi}}{\partial \tilde{x}}, \\
\frac{\partial \tilde{u}_{i y}}{\partial \tilde{t}}+\left(\tilde{u}_{i x} \frac{\partial}{\partial \tilde{x}}+\tilde{u}_{i y} \frac{\partial}{\partial \tilde{y}}\right) \tilde{u}_{i y}=-\frac{\partial \tilde{\phi}}{\partial \tilde{y}}+\Omega \tilde{u}_{i z},
\end{array}
$$




$$
\begin{array}{r}
\frac{\partial \tilde{u}_{i z}}{\partial \tilde{t}}+\left(\tilde{u}_{i x} \frac{\partial}{\partial \tilde{x}}+\tilde{u}_{i y} \frac{\partial}{\partial \tilde{y}}\right) \tilde{u}_{i z}=-\Omega \tilde{u}_{i y}, \\
0=\tilde{\nabla} \tilde{\phi}-\frac{\operatorname{Li}_{1 / 2}\left(-e^{\beta \mu_{0}}\right)}{\operatorname{Li}_{1 / 2}\left(-e^{\beta \mu}\right)} \tilde{\nabla} \tilde{n}_{e} \\
+\frac{H^{2}}{2} \tilde{\nabla}\left[\frac{1}{\sqrt{\tilde{n}_{e}}}\left(\frac{\partial^{2}}{\partial \tilde{x}^{2}}+\frac{\partial^{2}}{\partial \tilde{y}^{2}}\right) \sqrt{\tilde{n}_{e}}\right], \\
\left(\frac{\partial^{2}}{\partial \tilde{x}^{2}}+\frac{\partial^{2}}{\partial \tilde{y}^{2}}\right) \tilde{\phi}=\tilde{n}_{e}-\tilde{n}_{i},
\end{array}
$$

where $\Omega=\omega_{c i} / \omega_{p i}$ has been defined and where $\tilde{\nabla}=$ $(\partial / \partial \tilde{x}, \partial / \partial \tilde{y}, 0)$, while Eq. (8) becomes

$$
\tilde{n}_{e}=\frac{\mathrm{Li}_{3 / 2}\left(-e^{\beta \mu}\right)}{\mathrm{Li}_{3 / 2}\left(-e^{\beta \mu_{0}}\right)} .
$$

In the following calculations, for brevity the tilde sign used for defining normalized quantities is omitted.

In order to find a nonlinear evolution equation describing the magnetized plasma, the stretching of the independent variables $x, y$, and $t$ is defined under the assumption of strong magnetization as [41-44]

$$
X=\varepsilon^{1 / 2}\left(x-V_{0} t\right), \quad Y=\varepsilon^{1 / 2} y, \quad \tau=\varepsilon^{3 / 2} t,
$$

where $\varepsilon$ is a formal small expansion parameter and $V_{0}$ is the phase velocity of the wave, to be determined later. The perturbed quantities can be expanded in powers of $\varepsilon$ as follows:

$$
\begin{aligned}
n_{j} & =1+\varepsilon n_{j 1}+\varepsilon^{2} n_{j 2}+\ldots, \quad j=e, i, \\
u_{i x} & =\varepsilon u_{x 1}+\varepsilon^{2} u_{x 2}+\varepsilon^{3} u_{x 3} \ldots, \\
u_{i \perp} & =\varepsilon^{3 / 2} u_{\perp 1}+\varepsilon^{2} u_{\perp 2}+\varepsilon^{5 / 2} u_{\perp 3} \ldots, \quad \perp=y, z, \\
\phi & =\varepsilon \phi_{1}+\varepsilon^{2} \phi_{2}+\cdots, \\
\mu & =\mu_{0}+\varepsilon \mu_{1}+\varepsilon^{2} \mu_{2}+\cdots .
\end{aligned}
$$

In the present model, the ion velocity components $\left(u_{i y}, u_{i z}\right)$ perpendicular to the magnetic-field directions are taken as higher order perturbations compared to the parallel component $u_{i x}$ since, in the presence of a strong magnetic field, the plasma is anisotropic so that the ion gyromotion becomes a higher order effect.

The lowest $\varepsilon$-order terms $\left(\sim \varepsilon^{3 / 2}\right)$ from the set of Eqs. (38)(42) give

$$
\begin{array}{r}
-V_{0} \frac{\partial n_{i 1}}{\partial X}+\frac{\partial u_{x 1}}{\partial X}=0, \\
V_{0} \frac{\partial u_{x 1}}{\partial X}=\frac{\partial \phi_{1}}{\partial X}, \\
u_{z 1}=\frac{1}{\Omega} \frac{\partial \phi_{1}}{\partial Y}, \\
-\Omega u_{y 1}=0, \\
\frac{\partial \phi_{1}}{\partial X}=\frac{\partial n_{e 1}}{\partial X} .
\end{array}
$$

The velocity $u_{z 1}$ appears in Eq. (53) due to the $E \times B$ drift.

The lowest $\varepsilon$-order terms $(\sim \varepsilon)$ from Eqs. (43) and (44) give

$$
n_{i 1}=n_{e 1}=\frac{\mathrm{Li}_{1 / 2}\left(-e^{\beta \mu_{0}}\right)}{\mathrm{Li}_{3 / 2}\left(-e^{\beta \mu_{0}}\right)} \beta \mu_{1} \text {. }
$$

Solving the system (51)-(56), we get $V_{0}= \pm 1$. We set $V_{0}=1$ (the normalized phase velocity of the MIAW) without loss of generality.

Collecting the next higher order terms of the ion continuity $\left(\sim \varepsilon^{5 / 2}\right)$ and of the $X, Y$, and $Z$ components of the ion momentum equations $\left(\sim \varepsilon^{5 / 2}, \varepsilon^{2}, \varepsilon^{2}\right)$, and after rearrangement, we find

$$
\begin{array}{r}
\frac{\partial n_{i 1}}{\partial \tau}-\frac{\partial n_{i 2}}{\partial X}+\frac{\partial u_{x 2}}{\partial X}+\frac{\partial}{\partial X}\left(n_{i 1} u_{x 1}\right)+\frac{\partial u_{y 2}}{\partial Y}=0, \\
\frac{\partial u_{x 1}}{\partial \tau}-\frac{\partial u_{x 2}}{\partial X}+u_{x 1} \frac{\partial}{\partial X} u_{x 1}=-\frac{\partial \phi_{2}}{\partial X}, \\
-\frac{\partial u_{y 1}}{\partial X}=\Omega u_{z 2}, \\
\frac{\partial u_{z 1}}{\partial X}=\Omega u_{y 2} .
\end{array}
$$

Using Eq. (56) and the next higher order terms $\sim \varepsilon^{5 / 2}$ from the equations of motion of the inertialess degenerate electrons in the $X$ and $Y$ directions, we get

$\frac{\partial n_{e 2}}{\partial X}=\frac{\partial \phi_{2}}{\partial X}+\alpha n_{e 1} \frac{\partial n_{e 1}}{\partial X}+\frac{H^{2}}{4}\left(\frac{\partial^{3}}{\partial X^{3}}+\frac{\partial}{\partial X} \frac{\partial^{2}}{\partial Y^{2}}\right) n_{e 1}$

and

$\frac{\partial n_{e 2}}{\partial Y}=\frac{\partial \phi_{2}}{\partial Y}+\alpha n_{e 1} \frac{\partial n_{e 1}}{\partial Y}+\frac{H^{2}}{4}\left(\frac{\partial}{\partial Y} \frac{\partial^{2}}{\partial X^{2}}+\frac{\partial^{3}}{\partial Y^{3}}\right) n_{e 1}$,

where $\alpha$ has been defined in Eq. (12).

Now collecting the $\varepsilon^{2}$-order terms from Poisson's equation, we have

$$
\left(\frac{\partial^{2}}{\partial X^{2}}+\frac{\partial^{2}}{\partial Y^{2}}\right) \phi_{1}=n_{e 2}-n_{i 2}
$$

while the next-higher terms from Eqs. (57), (58), and (60) give

$$
\begin{aligned}
\frac{\partial n_{i 2}}{\partial X}= & \frac{\partial n_{i 1}}{\partial \tau}+\frac{\partial}{\partial X}\left(n_{i 1} u_{x 1}\right)+\frac{1}{\Omega} \frac{\partial}{\partial Y}\left(\frac{\partial u_{z 1}}{\partial X}\right) \\
& +\frac{\partial u_{x 1}}{\partial \tau}+u_{x 1} \frac{\partial u_{x 1}}{\partial X}+\frac{\partial \phi_{2}}{\partial X} .
\end{aligned}
$$

Differentiating Eq. (63) with respect to $X$ and using Eqs. (61) and (64) together with $n_{i 1}=n_{e 1}=u_{x 1}=\phi_{1}, u_{z 1}=$ $(1 / \Omega) \partial \phi_{1} / \partial Y$, it is finally possible to write the ZK equation for obliquely propagating quantum MIAWs in terms of $\phi_{1} \equiv \varphi$ :

$$
\frac{\partial \varphi}{\partial \tau}+A \varphi \frac{\partial \varphi}{\partial X}+\frac{\partial}{\partial X}\left(B \frac{\partial^{2} \varphi}{\partial X^{2}}+C \frac{\partial^{2} \varphi}{\partial Y^{2}}\right)=0 .
$$

The nonlinearity coefficient $A$ and the dispersion coefficients $B$ and $C$, in the parallel and perpendicular directions of the magnetic field, respectively, are defined as

$$
\begin{array}{r}
A=\frac{1}{2}(3-\alpha), \\
B=\frac{1}{2}\left(1-\frac{H^{2}}{4}\right), \\
C=\frac{1}{2}\left(1+\frac{1}{\Omega^{2}}-\frac{H^{2}}{4}\right) .
\end{array}
$$


In the purely classical limit the nonlinearity and dispersion coefficients become $A=1, B=1 / 2$, and $C=(1 / 2)$ $\left(1+1 / \Omega^{2}\right)$, in agreement with Refs. [17,43] and [44], treating MIAWs in a classical electron-ion plasma. In addition, the ZK equation for fully degenerate plasma will have $A=$ $4 / 3$ and $H=(1 / 2) \hbar \omega_{p e} / E_{F}$ in coefficients $B$ and $C$. The associated fully degenerate $\mathrm{ZK}$ equation does not match the results from Refs. [20-22], after comparison using physical (dimensional and nonstretched) coordinates, restricted to the case of electron-ion plasmas. Note that the ZK equations from the previous works do not match the purely classical result.

Provided $l_{x}^{2} B+l_{y}^{2} C \neq 0$, the soliton solution of the $\mathrm{ZK}$ equation (65), for obliquely propagating MIAWs is given by

$$
\varphi=\varphi_{0} \operatorname{sech}^{2}(\eta / W)
$$

where $\varphi_{0}=3 u_{0} /\left(A l_{x}\right)$ is the height and $W=$ $\sqrt{4 l_{x}\left(l_{x}^{2} B+l_{y}^{2} C\right) / u_{0}}$ is the width of the soliton in terms of the stretched coordinates. The polarity of the soliton depends on the sign of $\varphi_{0}$. The transformed coordinate $\eta$ in the comoving frame is defined as $\eta=l_{x} X+l_{y} Y-u_{0} \tau$, where $u_{0} \neq 0$ is the speed of the nonlinear pulse and $l_{x}>0$ and $l_{y}$ are direction cosines, so that $l_{x}^{2}+l_{y}^{2}=1$. To obtain localized structures, decaying boundary conditions as $\eta \rightarrow \pm \infty$ were applied. Following the habitual usage the term "soliton" is applied to the solitary wave (69), although the ZK equation does not belong to the class of completely integrable evolution equations. The dispersion effects arising from the combination of charge separation and finite ion Larmor radius balance the nonlinearity in the system to form the soliton.

Defining $\delta V=\varepsilon u_{0} / l_{x}$, in the laboratory frame the solution reads

$$
\begin{aligned}
\varphi= & \frac{3 \delta V}{A} \operatorname{sech}^{2}\left\{\frac{1}{2}\left(\frac{\delta V}{l_{x}^{2} B+l_{y}^{2} C}\right)^{1 / 2}\right. \\
& \left.\times\left[l_{x}\left(x-\left(V_{0}+\delta V\right) t\right)+l_{y} y\right]\right\} .
\end{aligned}
$$

It is apparent that $V_{0}+\delta V$ corresponds to the velocity at which the intersection between a plane of constant phase and a field line travels, down the same field line [42].

From Eq. (70) the width $L$ of the soliton in the laboratory frame is

$$
L=2\left(\frac{l_{x}^{2} B+l_{y}^{2} C}{\delta V}\right)^{1 / 2}=\sqrt{\frac{2}{\delta V}}\left(1-\frac{H^{2}}{4}+\frac{l_{y}^{2}}{\Omega^{2}}\right)^{1 / 2} .
$$

From this expression one concludes that while in the nonquantum $H=0$ limit necessarily $\delta V>0$ (bright soliton propagating at supersonic speed), in the quantum case one has the theoretical possibility of $\delta V<0$ (dark soliton propagating at subsonic speed), provided $H^{2} / 4>1+l_{y}^{2} / \Omega^{2}$.

It is interesting to single out the different quantum effects in the soliton (70). The amplitude/dip of bright/dark solitons is inversely proportional to $A$, which depends only on quantum degeneracy, and not on quantum diffraction. More degenerate systems produce a smaller $\alpha$-as shown, e.g., in Fig. 1-and hence a larger $A$. Therefore the soliton amplitude/dip decreases with the degeneracy. On the other hand, from the width (71), one finds the dependence on the quantum diffraction parameter $H^{2}$ (which is also dependent on the degree of degeneracy)

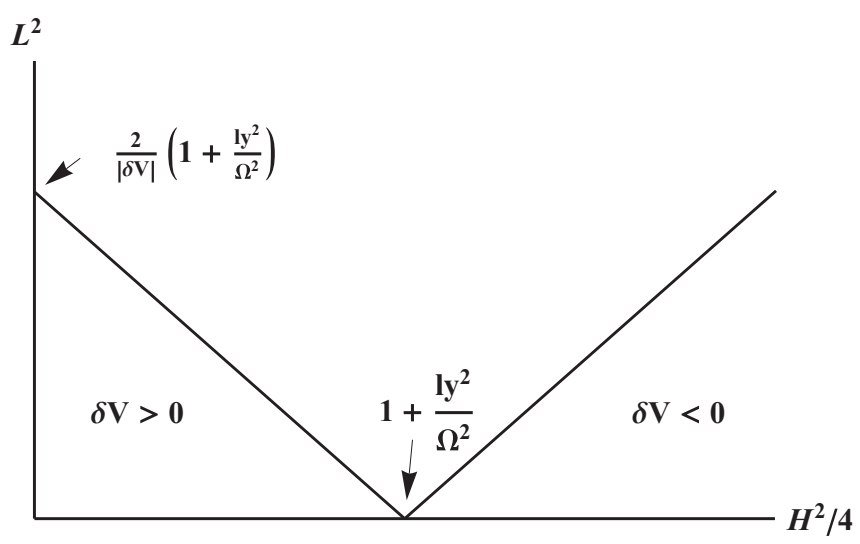

FIG. 3. Squared width of the localized structure as shown in Eq. (71), as a function of the quantum diffraction parameter $H^{2}$.

shown in Fig. 3. For semiclassical bright solitons $(\delta V>0)$ one has $L^{2}$ decreasing with quantum effects, while for quantum dark solitons $(\delta V<0)$ one has $L^{2}$ increasing with quantum effects.

In unmagnetized quantum plasmas, by construction the term $\sim 1 / \Omega^{2}$ does not appear. In this case when $H=2$ the corresponding Korteweg-de Vries (KdV) equation collapses to Burger's equation, producing an ion-acoustic shock wave structure instead of a soliton [8].

In the magnetized case, a further possibility occurs when $C=0$, or, equivalently, $1+1 / \Omega^{2}=H^{2} / 4$, which is not allowed in the classical limit $(H \equiv 0)$. When $C=0$, from Eq. (65) one has

$$
\frac{\partial \varphi}{\partial \tau}+A \varphi \frac{\partial \varphi}{\partial X}-\frac{1}{2 \Omega^{2}} \frac{\partial^{3} \varphi}{\partial X^{3}}=0,
$$

which transforms to the $\mathrm{KdV}$ equation in its standard form by means of $\varphi \rightarrow-\varphi, X \rightarrow-X, \tau \rightarrow \tau$. Therefore, in this particular situation the problem becomes completely integrable.

Finally, if $B=0$, it means that $1-H^{2} / 4=0$, due to which $C=1 / 2 \Omega^{2}$, so that Eq. (65) becomes

$$
\frac{\partial \varphi}{\partial \tau}+A \varphi \frac{\partial \varphi}{\partial X}+\frac{1}{2 \Omega^{2}} \frac{\partial}{\partial X} \frac{\partial^{2} \varphi}{\partial Y^{2}}=0 .
$$

This is a KdV-like equation having dispersion effects perpendicular to the magnetic field, due to the obliquely propagating MIAW.

Regarding the ranges of validity of the parameters $A, B$, and $C$ in Eqs. (66)-(68), first we observe that from Eq. (12) one has $1 / 3<\alpha<1$, so that $1<A<4 / 3$. In addition, since $H^{2}$ from Eq. (23) can, in principle, attain any non-negative value, $B$ and $C$ are not positive definite. However, strictly speaking, very large values of $H^{2}$ are associated with strongly coupling effects, which can have a large impact on soliton propagation, to be addressed in a separate extended theory. Only in such a generalized framework could one be able to make more precise statements on the existence or nonexistence of pure quantum solitons. Nevertheless, significant values of $H^{2}$ are certainly physically acceptable, as found from the present treatment. These issues are discussed in the following section. 


\section{APPLICATIONS}

It is important to discuss the validity domain of the general theory devised in the last sections. Moreover, it is highly desirable to offer precise physical parameters where the predicted linear and nonlinear waves could be searched for in practice. Obviously, the theory is more relevant in the intermediate regimes, where the thermal and Fermi temperatures are not significantly different. Otherwise, the fully degenerate or dilute limits could be sufficiently accurate. Therefore, in this section we frequently assume that

$$
T=T_{F},
$$

where $T_{F}=E_{F} / \kappa_{B}$ is the electron's Fermi temperature.

To start, consider the normalization condition (9), which can be expressed [26] as

$$
\mathrm{Li}_{3 / 2}(-z)=-\frac{4}{3 \sqrt{\pi}}\left(\beta E_{F}\right)^{3 / 2},
$$

where $z=\exp \left(\beta \mu_{0}\right)$. For equal thermal and Fermi temperatures, $\beta E_{F}=1$, which from Eq. (75) gives the equilibrium fugacity $z=0.98$ and from Eq. (12) a parameter $\alpha=0.80$, definitely in the intermediate dilute-degenerate situation as explicitly shown, e.g., in Fig. 1.

Besides quantum degeneracy, quantum diffraction effects can also provide qualitatively new aspects as found, e.g., in the extra dispersion of linear waves in Sec. III and the modified width of the solitons in Sec. VI. Therefore it would be interesting to investigate systems with a large parameter $H$. However, realistically speaking it is not possible to increase $H$ without limits, which would enter the strongly coupled plasma regime, not included in the present formalism. For instance, the ideal Fermi gas equation of state for electrons would be unappropriated. Therefore, it is necessary to analyze the coupling parameter $g$ for electrons, which can be defined [45] as $g=l / a$, where

$$
l=-\frac{e^{2}}{12 \pi \varepsilon_{0} \kappa_{B} T} \frac{n_{0} \Lambda_{T}^{3}}{\operatorname{Li}_{5 / 2}(-z)}
$$

is a generalized Landau length involving the thermal de Broglie wavelength $\Lambda_{T}=\left[2 \pi \hbar^{2} /\left(m_{e} \kappa_{B} T\right)\right]^{1 / 2}$, and $a=$ $\left(4 \pi n_{0} / 3\right)^{-1 / 3}$ is the Wigner-Seitz radius. In the dilute case, one has $e^{2} /\left(4 \pi \varepsilon_{0} l\right)=(3 / 2) \kappa_{B} T$, so that $l$ would be the classical distance of closest approach in a binary collision, for average kinetic energy. The general expression (76), accounts for the degeneracy effects on the mean kinetic energy. A few calculations yield

$$
g=-\frac{2 \alpha_{F} \sqrt{2 \beta m_{e} c^{2}}}{3(3 \sqrt{\pi})^{1 / 3}} \frac{\left[\mathrm{Li}_{3 / 2}^{2}(-z)\right]^{2 / 3}}{\mathrm{Li}_{5 / 2}(-z)},
$$

an expression similar to the one for $H^{2}$ in Eq. (23). Hence, it is legitimate to suspect that the indiscriminate increase in quantum diffraction gives rise to nonideality effects such as dynamical screening and bound states [45]. Incidentally Eq. (77) agrees with Eq. (16) of [29], found from related but different methods.

The resemblance between the coupling and the quantum diffraction parameters is confirmed in Fig. 4, which can be compared to Fig. 2 for $H^{2}$. Moreover, it is apparent in Fig. 5 that we have $H^{2}<0.5$ for the whole span of degeneracy

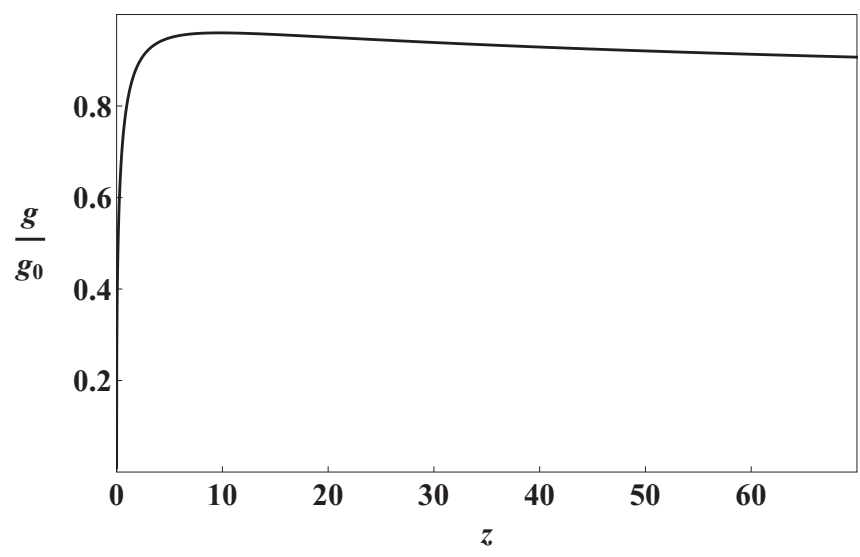

FIG. 4. Coupling parameter defined in Eq. (77), as a function of the equilibrium fugacity $z=e^{\beta \mu_{0}}$, and normalized to $g_{0}=$ $2 \alpha_{F} \sqrt{2 \beta m_{e} c^{2}} /\left[3(3 \sqrt{\pi})^{1 / 3}\right]$.

regimes, as far as $g<1$. Strictly speaking, the dark soliton, shock wave, and completely integrable case associated with the $\mathrm{KdV}$ equation (72), are therefore outside the validity domain of the model, since they require large values of the quantum diffraction parameter $H$. Nevertheless, the influence of the wave nature of the electrons can still provide important corrections on its own, at least for reasonable values of $H^{2}$, as is obvious, for instance, in the width of the ZK soliton in Eq. (71).

The behavior of the parameters $g$ and $H^{2}$ is summarized in Fig. 6 for $T=T_{F}$ and considering hydrogen plasma parameters. We observe that $g<1$ for $n_{0}>5.23 \times 10^{28} \mathrm{~m}^{-3}$, or $E_{F}>5.11 \mathrm{eV}$, which starts becoming realizable for typical densities in solid-density plasmas [46,47].

It is necessary to discuss additional points about the validity conditions of the model. Both the static electronic response and the long-wavelength (and hence fluid) assumptions are collected in Eq. (27) of [29], reproduced here for convenience:

$$
k_{\min } \equiv \frac{2 \sqrt{3} m_{e} c_{s}}{\hbar} \ll k \ll \frac{\omega_{p i}}{c_{s}} \equiv k_{\max } .
$$

For hydrogen plasma and $T=T_{F}$, from Eq. (78) one has $k_{\max }>k_{\min }$ for $n_{0}<9.81 \times 10^{35} \mathrm{~m}^{-3}$. The latter condition is

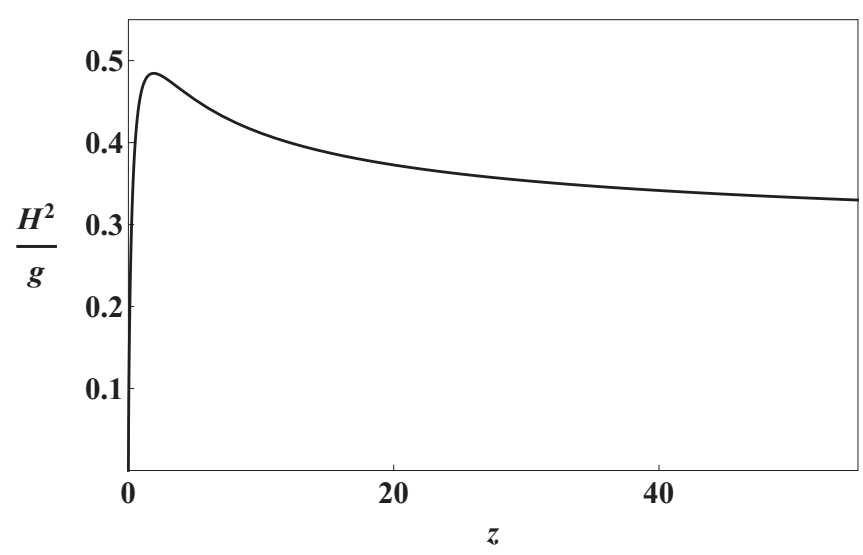

FIG. 5. Ratio between the diffraction parameter $H^{2}$ from Eq. (23) and the coupling parameter $g$ from Eq. (77), as a function of the equilibrium fugacity $z=e^{\beta \mu_{0}}$. 


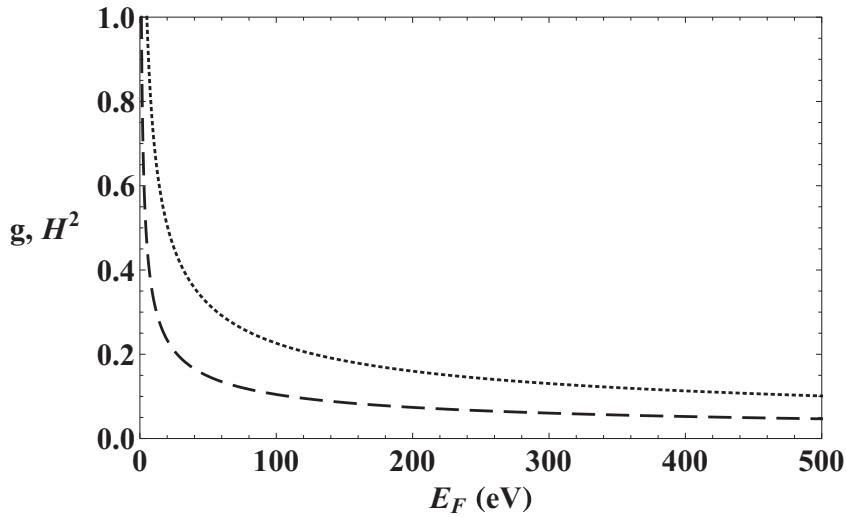

FIG. 6. Upper, dotted curve: Coupling parameter $g$ from Eq. (77), Lower, dashed curve: Quantum diffraction parameter $H^{2}$ from Eq. (23), as a function of the electronic Fermi energy $E_{F}$ (in eV), for hydrogen plasma and the intermediate dilute-degenerate regime where $T=T_{F}$.

safely satisfied for nonrelativistic plasma. At the high densities available in compact astrophysical objects like white dwarfs and neutron stars, the Fermi momentum becomes comparable to $m_{e} c$, requiring a relativistic treatment. Strictly speaking, the first inequality in Eq. (78) could be removed, but in this case the quantum recoil would be less significant. In this case, only quantum degeneracy effects would be relevant.

In terms of the wavelength $\lambda$, Eq. (78) yields a suitable range, $\lambda_{\min }=2 \pi / k_{\max } \ll \lambda \ll \lambda_{\max }=2 \pi / k_{\min }$, shown in Fig. 7, on the nanometric scale from extreme ultraviolet to soft $\mathrm{x}$ rays.

As discussed in Sec. IV, the model also assumes that the pressure effects are significantly larger than the magnetic-field effects regarding electrons, or $k^{2} v_{T}^{2}(k) \gg \omega_{c e}^{2}$. In the worst case, where $k \approx k_{\min }$, the quantum diffraction is typically a correction in expression (30) for $v_{T}(k)$. We then find

$$
\frac{\hbar \omega_{c e}}{E_{F}} \ll 2 \sqrt{3}\left(\frac{m_{e}}{m_{i}}\right)^{1 / 2} \frac{\mathrm{Li}_{3 / 2}(-z)}{\mathrm{Li}_{1 / 2}(-\mathrm{z})} \approx 0.10
$$

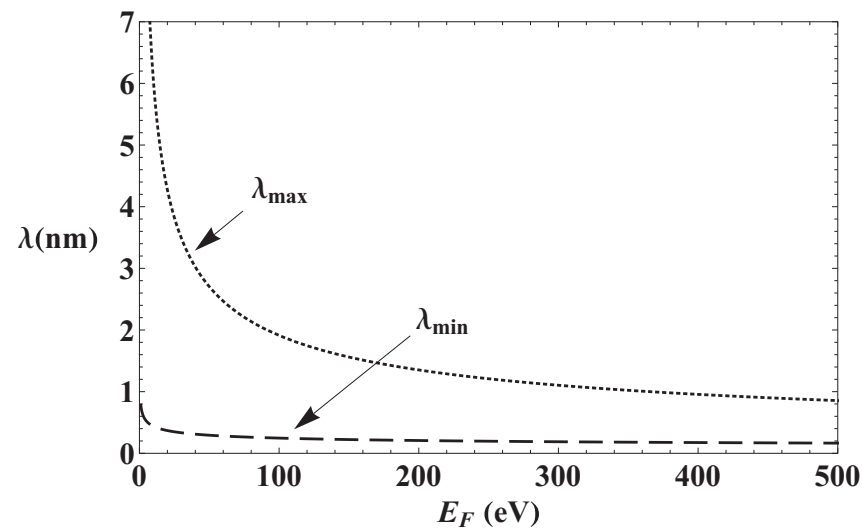

FIG. 7. Upper, dotted curve: Maximum wavelength $\lambda_{\max }$. Lower, dashed curve: Minimal wavelength $\lambda_{\min }$, consistent with Eq. (78), as a function of the electronic Fermi energy $E_{F}$ (in $\mathrm{eV}$ ), for hydrogen plasma and the intermediate dilute-degenerate regime where $T=T_{F}$.

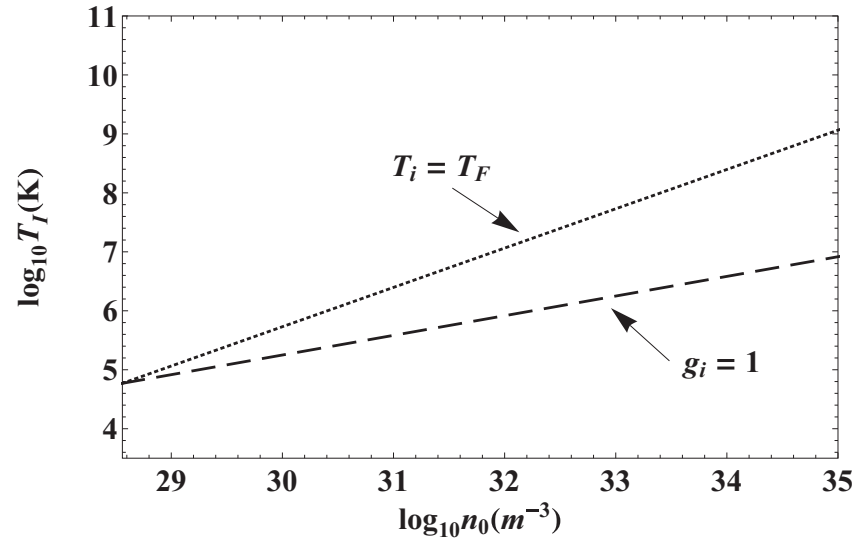

FIG. 8. Cold and weakly coupled ions are below the upper, dotted straight line (where the ionic temperature $T_{i}$ equals the electronic Fermi temperature $T_{F}$ ) and above the lower, dashed straight line (where the ionic coupling parameter $g_{i}=1$ ).

for $T=T_{F}$. Condition (79), which is easier to satisfy for higher densities, is safely satisfied except for very strong magnetic fields. For instance, for $E_{F} \approx 10 \mathrm{eV}$, we just need $B_{0} \ll 8.81 \mathrm{kT} a$.

Ions have been assumed to be cold, classical, and ideal (disregarding strong ion coupling effects). Denoting $T_{i}$ the ionic temperature, when $T_{F} \gg T_{i}$ the MIAW phase speed becomes much higher than the ionic thermal speed, justifying the cold-ion assumption. On the other hand, there is the need for a small ionic coupling parameter $g_{i}$. For a nondegenerate ionic fluid we then [45] have

$$
g_{i}=\frac{e^{2}}{4 \pi \varepsilon_{0} a}\left(\frac{3 \kappa_{B} T_{i}}{2}\right)^{-1} \ll 1 .
$$

Otherwise, one would have an ionic liquid or even an ionic crystal, as is believed to happen for $g_{i} \approx 172$ in a onecomponent plasma [48]. The joint requirements of cold and non-strongly coupled ions are represented in Fig. 8, where the

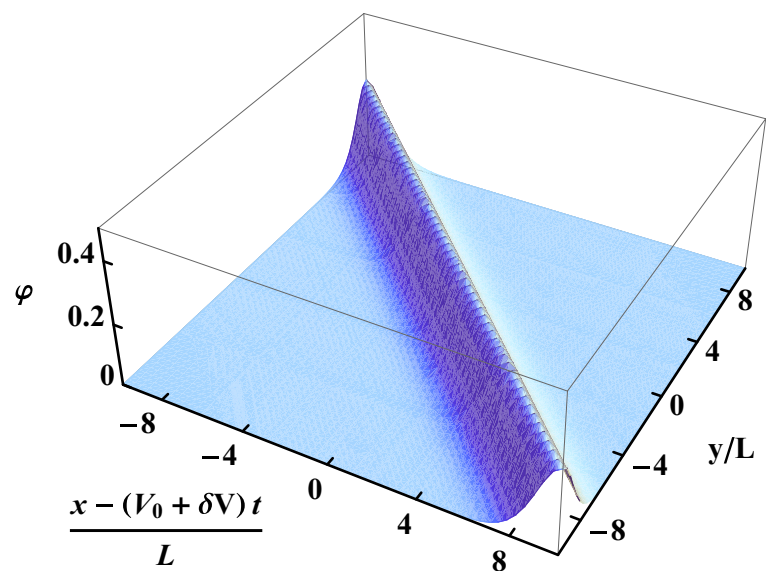

FIG. 9. Two-dimensional profile of the MIAW hump soliton structure (70), moving at supersonic speed in the laboratory frame, using normalized coordinates. Parameters: $n_{0}=5 \times 10^{30} \mathrm{~m}^{-3}, H^{2}=$ $0.10, g=0.22, T=T_{F}=1.24 \times 10^{6} \mathrm{~K}, B_{0}=10^{3} \mathrm{~T}, l_{x}=l_{y}=$ $\sqrt{2} / 2$, and $\delta V=0.10$. 
allowable region is between the straight lines, $T_{i}=T_{F}, g_{i}=1$. A minimal number density, $n_{0}=3.63 \times 10^{28} \mathrm{~m}^{-3}$, is found to be necessary, which again is accessible for typical solid-density plasmas.

For the sake of illustration, consider the bright soliton solution from Eq. (70), for parameters representative of soliddensity plasmas, namely, $n_{0}=5 \times 10^{30} \mathrm{~m}^{-3}, H^{2}=0.10, g=$ 0.22 , and $T=T_{F}=1.24 \times 10^{6} \mathrm{~K}$. In this case the allowable wave numbers satisfy $0.24 \mathrm{~nm}<\lambda<1.85 \mathrm{~nm}$, the ionic temperatures are in the range $3.04 \times 10^{5} \mathrm{~K}<T_{i}<1.57 \times 10^{6}$ $\mathrm{K}$, and the magnetic field should have a strength $B_{0}<$ $9.23 \times 10^{4} \mathrm{~T}$. The soliton is shown in Fig. 9, where $B_{0}=10^{3}$ $\mathrm{T}, l_{x}=l_{y}=\sqrt{2} / 2$, and $\delta V=0.10$.

\section{SUMMARY}

The main results of this work are the dispersion relation (18), and the ZK equation (65), both of which describe quantum MIAWs in the linear and nonlinear regimes, respectively, allowing for an arbitrary electron degeneracy degree. The results significantly generalize the previous literature, restricted to either dilute (Maxwell-Boltzmann) or fully degenerate plasmas. The conditions for applications are investigated in depth, pointing out the importance of the findings for, e.g., magnetized solid-density plasma. While the physical parameters have been more focused on hydrogen plasma in the intermediate dilute-degenerate limit where $T=T_{F}$, adaptation to fully ionized electron-ion cases with atomic number $Z \neq 1$ and arbitrary temperatures is not difficult at all. With minor adjustments, the whole parametric analysis in Sec. VII also applies to the unmagnetized case. It is hoped that the detailed assessment of physical parameters thus developed, will incentive experimental and observational verifications of linear and nonlinear quantum ion-acoustic waves, both in the laboratory and in nature, considering a large span of degeneracy regimes. A possibly important next step is the rigorous (non-ad hoc) incorporation of exchange-correlation effects, which are beyond the scope of the present paper.

\section{ACKNOWLEDGMENT}

F.H. acknowledges Conselho Nacional de Desenvolvimento Científico e Tecnológico $(\mathrm{CNPq})$ for financial support.

\section{APPENDIX: DERIVATION OF EQ. (36)}

From Eqs. (34) and (35) one has

$$
\begin{aligned}
\chi_{e}(0, \mathbf{k}) & =\frac{\mathcal{A} e^{2}}{\varepsilon_{0} \hbar k^{2}} \int d \mathbf{v}\left[\frac{1}{\mathbf{k} \cdot \mathbf{v}+\hbar k^{2} /\left(2 m_{e}\right)}-\frac{1}{\mathbf{k} \cdot \mathbf{v}-\hbar k^{2} /\left(2 m_{e}\right)}\right] \frac{1}{1+\exp \left(\beta m_{e} v^{2} / 2\right) / z} \\
& =\frac{2 \pi \mathcal{A} e^{2}}{\varepsilon_{0} \hbar k^{2}} \int_{-\infty}^{\infty} d v_{\|}\left[\frac{1}{k v_{\|}+\hbar k^{2} /\left(2 m_{e}\right)}-\frac{1}{k v_{\|}-\hbar k^{2} /\left(2 m_{e}\right)}\right] \int_{0}^{\infty} d v_{\perp} \frac{v_{\perp}}{1+\exp \left[\beta m_{e}\left(v_{\perp}^{2}+v_{\|}^{2}\right) / 2\right] / z},
\end{aligned}
$$

where $\mathbf{v}=v_{\|} \mathbf{k} / k+\mathbf{v}_{\perp}, \mathbf{k} \cdot \mathbf{v}_{\perp}=0, z=\exp \left(\beta \mu_{0}\right)$, and all integrals consider the principal value sense.

Performing the $v_{\perp}$ integral, considering the expression of $\mathcal{A}$ in Eq. (9), and applying a simple change of variables, we get

$$
\chi_{e}(0, \mathbf{k})=\frac{\beta m_{e} \omega_{p e}^{2}}{4 \sqrt{\pi} \mathrm{Li}_{3 / 2}(-z) q k^{2}} \int_{-\infty}^{\infty} \frac{d s}{s}\left[\ln \left(1+z e^{-(s+q)^{2}}\right)-\ln \left(1+z e^{-(s-q)^{2}}\right)\right],
$$

where $q=\sqrt{\beta /\left(2 m_{e}\right)} \hbar k / 2$.

Expanding in powers of the quantum recoil one has

$$
\ln \left(1+z e^{-(s+q)^{2}}\right)-\ln \left(1+z e^{-(s-q)^{2}}\right)=-4 q\left(g(s)+\frac{q^{2}}{3 !} g^{\prime \prime}(s)+\frac{q^{4}}{5 !} g^{(i v)}(s)+O\left(q^{6}\right)\right),
$$

where $g(s) \equiv s /\left[1+\exp \left(s^{2}\right) / z\right]$. At this point, note that the possible divergence at $s=0$ in integral (A2) has been explicitly removed.

After integrating by parts, it is found that

$$
\chi_{e}(0, \mathbf{k})=-\frac{2 \beta m_{e} \omega_{p e}^{2} z}{\sqrt{\pi} \operatorname{Li}_{3 / 2}(-z) k^{2}}\left(\int_{0}^{\infty} \frac{d s}{z+e^{s^{2}}}-\frac{2 q^{2}}{3} \int_{0}^{\infty} \frac{d s e^{s^{2}}}{\left(z+e^{s^{2}}\right)^{2}}+\frac{4 q^{4}}{15} \int_{0}^{\infty} \frac{d s e^{s^{2}}\left(e^{s^{2}}-z\right)}{\left(z+e^{s^{2}}\right)^{3}}+O\left(q^{6}\right)\right) .
$$

From Eqs. (10) and (11), each term on the right-hand side of (A4) can be evaluated in terms of polylogarithms. For instance,

$$
\begin{aligned}
\operatorname{Li}_{1 / 2}(-z) & =-\frac{2 z}{\sqrt{\pi}} \int_{0}^{\infty} \frac{d s}{z+e^{s^{2}}}, \\
\mathrm{Li}_{-1 / 2}(-z) & =-\frac{2 z}{\sqrt{\pi}} \int_{0}^{\infty} \frac{d s e^{s^{2}}}{\left(z+e^{s^{2}}\right)^{2}}, \\
\mathrm{Li}_{-3 / 2}(-z) & =\frac{2 z}{\sqrt{\pi}} \int_{0}^{\infty} \frac{d s e^{s^{2}}\left(z-e^{s^{2}}\right)}{\left(z+e^{s^{2}}\right)^{3}},
\end{aligned}
$$

clearly related to the integrals in expression (A4). In this way Eq. (36), which is also independently confirmed by numerical evaluation, is proved. 
[1] P. A. Markowich, C. A. Ringhofer, and C. Schmeiser, Semiconductor Equations (Springer-Verlag, Vienna, 1990).

[2] M. Marklund and P. K. Shukla, Rev. Mod. Phys. 78, 591 (2006).

[3] S. H. Glenzer, O. L. Landen, P. Neumayer, R. W. Lee, K. Widmann, S. W. Pollaine, R. J. Wallace, G. Gregori, A. Höll, T. Bornath, R. Thiele, V. Schwarz, W.-D. Kraeft, and R. Redmer, Phys. Rev. Lett. 98, 065002 (2007).

[4] J. E. Cross, B. Reville, and G. Gregori, Astrophys. J. 795, 59 (2014).

[5] M. Opher, L. O. Silva, D. E. Dauger, V. K. Decyk, and J. M. Dawson, Phys. Plasmas 8, 2454 (2001).

[6] G. Chabrier, D. Saumon, and A. Y. Potekhin, J. Phys. A 39, 4411 (2006).

[7] A. K. Harding and D. Lai, Rep. Prog. Phys. 69, 2631 (2006).

[8] F. Haas, L. G. Garcia, J. Goedert, and G. Manfredi, Phys. Plasmas 10, 3858 (2003).

[9] F. Haas, Quantum Plasmas: A Hydrodynamic Approach (Springer, New York, 2011).

[10] R. E. Wyatt, Quantum Dynamics with Trajectories: Introduction to Quantum Hydrodynamics (Springer, New York, 2005).

[11] P. K. Shukla and B. Eliasson, Phys. Usp. 53, 51 (2010).

[12] F. Haas, Phys. Plasmas 12, 062117 (2005).

[13] H. Terças, J. T. Mendonca, and P. K. Shukla, Phys. Plasmas 15, 072109 (2008).

[14] J. Zamanian, M. Marklund, and G. Brodin, Phys. Rev. E 88, 063105 (2013).

[15] J. Zamanian, M. Marklund, and G. Brodin, Eur. Phys. J. D 69, 25 (2015).

[16] R. Ekman, J. Zamanian, and G. Brodin, Phys. Rev. E 92, 013104 (2015).

[17] V. Zakharov and E. Kuznetsov, Sov. Phys. J. Exper. Theor. Phys. 39, 285 (1974).

[18] E. Infeld and G. Rowlands, Nonlinear Waves, Solitons and Chaos (Cambridge University Press, Cambridge, UK, 2000).

[19] E. W. Laedke and K. H. Spatschek, Phys. Rev. Lett. 47, 719 (1981).

[20] R. Sabry, W. M. Moslem, F. Haas. S. Ali, and P. K. Shukla, Phys. Plasmas 15, 122308 (2008).

[21] W. M. Moslem, S. Ali, P. K. Shukla, X. Y. Tang, and G. Rowlands, Phys. Plasmas 14, 082308 (2007).

[22] S. A. Khan and W. Masood, Phys. Plasmas 15, 062301 (2008).

[23] N. Maafa, Phys. Scr. 48, 351 (1993).

[24] A. E. Dubinov, A. A. Dubinova, and M. A. Sazokin, J. Commun. Technol. Electron. 55, 907 (2010).
[25] A. E. Dubinov and I. N. Kitaev, Phys. Plasmas 21, 102105 (2014).

[26] B. Eliasson and P. K. Shukla, Phys. Scr. 78, 025503 (2008).

[27] M. McKerr, F. Haas, and I. Kourakis, Phys. Rev. E 90, 033112 (2014).

[28] E. E. Behery, F. Haas, and I. Kourakis, Phys. Rev. E 93, 023206 (2016).

[29] F. Haas and S. Mahmood, Phys. Rev. E 92, 053112 (2015).

[30] V. E. Fortov, Extreme States of Matter on Earth and in the Cosmos (Springer, Berlin, 2011).

[31] R. K. Pathria and P. D. Beale, Statistical Mechanics, 3rd ed. (Elsevier, New York, 2011).

[32] L. Lewin, Polylogarithms and Associated Functions (North-Holland, New York, 1981).

[33] B. Eliasson and M. Akbari-Moghanjoughi, Phys. Lett. A 380, 2518 (2016).

[34] L. G. Stanton and M. S. Murillo, Phys. Rev. E 91, 033104 (2015).

[35] L. G. Stanton and M. S. Murillo, Phys. Rev. E 91, 049901(E) (2015).

[36] T. E. Stringer, J. Nucl. Energy, Part C 5, 89 (1963).

[37] E. Witt and W. Lotko, Phys. Fluids 26, 2176 (1983).

[38] K. N. Stepanov, Sov. Phys. J. Exper. Theor. Phys. 8, 808 (1959).

[39] A. I. Akhiezer, I. A. Akhiezer, R. V. Polovin, A. G. Sitenko, and K. N. Stepanov, Plasma Electrodynamics (Pergamon Press, Oxford, UK, 1975), Vol. I.

[40] D. B. Melrose and A. Mushtaq, Phys. Rev. E 82, 056402 (2010).

[41] I. Kourakis, W. M. Moslem, U. M. Abdelsalam, R. Sabry, and P. K. Shukla, Plasma Fusion Res. 4, 018 (2009).

[42] R. L. Mace and M. A. Hellberg, Phys. Plasmas 8, 2649 (2001).

[43] E. Infeld, J. Plasma Phys. 33, 171 (1985).

[44] E. W. Laedke and K. H. Spatschek, Phys. Fluids 25, 985 (1982).

[45] D. Kremp, M. Schlanges, and W.-D. Kraeft, Quantum Statistics of Nonideal Plasmas (Springer-Verlag, Berlin, 2005).

[46] M. Tatarakis, I. Watts, F. N. Beg, E. L. Clark, A. E. Dangor, A. Gopal, M. G. Haines, P. A. Norreys, U. Wagner, M.-S. Wei, M. Zepf, and K. Krushelnick, Nature 415, 280 (2002).

[47] U. Wagner, M. Tatarakis, A. Gopal, F. N. Beg, E. L. Clark, A. E. Dangor, R. G. Evans, M. G. Haines, S. P. D. Mangles, P. A. Norreys, M.-S. Wei, M. Zepf, and K. Krushelnick, Phys. Rev. E 70, 026401 (2004).

[48] M. S. Murillo, Phys. Plasmas 11, 2964 (2004). 